\title{
Connectivity Properties of Horospheres in Euclidean Buildings and Applications to Finiteness Properties of Discrete Groups
}

\author{
Kai-Uwe Bux Kevin Wortman*
}

August 14, 2008

\begin{abstract}
Let $\mathbf{G}\left(\mathcal{O}_{S}\right)$ be an $S$-arithmetic subgroup of a connected, absolutely almost simple linear algebraic group $\mathbf{G}$ over a global function field $K$. We show that the sum of local ranks of $\mathbf{G}$ determines the homological finiteness properties of $\mathbf{G}\left(\mathcal{O}_{S}\right)$ provided the $K$-rank of $\mathbf{G}$ is 1 . This shows that the general upper bound for the finiteness length of $\mathbf{G}\left(\mathcal{O}_{S}\right)$ established in an earlier paper is sharp in this case.

The geometric analysis underlying our result determines the conectivity properties of horospheres in thick Euclidean buildings.
\end{abstract}

\section{Introduction}

Let $K$ be a global function field and suppose $\mathbf{G}$ is a connected, noncommutative, absolutely almost simple $K$-group. Let $S$ be a finite, nonempty set of pairwise inequivalent valuations on $K$. We let $\mathcal{O}_{S}$ be the ring of $S$-integers in $K$. We denote the completion of $K$ with respect to $v \in S$ by $K_{v}$. We let $k(\mathbf{G}, S)=\sum_{v \in S} \operatorname{rank}_{K_{v}}(\mathbf{G})$.

In [BW07], we proved:

Theorem 1.1. Suppose $K, S$, and $\mathbf{G}$ are as above. If $\operatorname{rank}_{K}(\mathbf{G})>0$, then $\mathbf{G}\left(\mathcal{O}_{S}\right)$ is not of type $F_{k(\mathbf{G}, S)}$.

\footnotetext{
*The second author was partially supported by NSF grant DMS - 0750032
} 
Recall that a group $\Gamma$ is of type $\mathrm{F}_{m}$ if there exists an Eilenberg-Mac Lane complex $K(\Gamma, 1)$ with finite $m$-skeleton. It follows that such a group has finitely generated homology and cohomology groups up to dimension $m$.

Theorem 1.1 leads to the natural question of whether the groups from Theorem 1.1 are of type $\mathrm{F}_{k(\mathbf{G}, S)-1}$. Several results show that for special cases, the answer is yes. For example, Stuhler [Stuh80] proved that the answer is yes for groups of the form $\mathrm{SL}_{2}\left(\mathcal{O}_{S}\right)$. And independent work of Abels [Abel91] and Abramenko Abra96. has shown that the answer is yes for some higher rank examples. In particular, the answer is yes if $\mathbf{G}$ is a classical $K_{q}$-group and $\mathcal{O}_{S}=\mathbb{F}_{q}[t]$ where $q$ is large depending on the rank of $\mathbf{G}$.

In this paper, we add to the evidence above by proving:

Theorem 1.2. Suppose $K, S$, and $\mathbf{G}$ are as in Theorem 1.1. If $\operatorname{rank}_{K}(\mathbf{G})=$ 1 , then $\mathbf{G}\left(\mathcal{O}_{S}\right)$ is of type $F_{k(\mathbf{G}, S)-1}$.

Thus, Theorem 1.2 is a generalization of the result of Stuhler mentioned above. And together with the higher rank examples including those of Abels and Abramenko, it seems reasonable to make the following:

Conjecture 1.3. Suppose $K, S$, and $\mathbf{G}$ are as in Theorem 1.1. If $\operatorname{rank}_{K}(\mathbf{G})>0$, then $\mathbf{G}\left(\mathcal{O}_{S}\right)$ is of type $F_{h(\mathbf{G}, S)-1}$.

\subsection{Background}

See the introduction of [BW07] for more on the background of this problem.

\subsection{Outline of the proof of Theorem 1.2}

The group $\mathbf{G}\left(\mathcal{O}_{S}\right)$ acts on the Euclidean building for $\prod_{v \in S} \mathbf{G}\left(K_{v}\right)$, which we denote by $X$. Since $\operatorname{rank}_{K}(\mathbf{G})=1>0$, the group $\mathbf{G}\left(\mathcal{O}_{S}\right)$ does not act cocompactly on $X$. Nor does $\mathbf{G}\left(\mathcal{O}_{S}\right)$, nor any finite index subgroup of $\mathbf{G}\left(\mathcal{O}_{S}\right)$, act freely on $X$, although $\mathrm{G}\left(\mathcal{O}_{S}\right)$ does act on $X$ with finite cell stabilizers.

We apply reduction theory to obtain a subspace $X_{0} \subseteq X$ on which $\mathbf{G}\left(\mathcal{O}_{S}\right)$ acts with compact quotient. The space $X_{0}$ is obtained from $X$ by removing an infinite family of pairwise disjoint horoballs.

We use piecewise linear Morse theory to prove that horospheres - the boundaries of horoballs - appearing in the construction of $X_{0}$ are $k(\mathbf{G}, S)-2$ connected. It follows that $X_{0}$ is $k(\mathbf{G}, S)-2$ connected, so $\mathbf{G}\left(\mathcal{O}_{S}\right)$ is of type $\mathrm{F}_{h(\mathbf{G}, S)-1}$. 
Our proof that the horospheres used to define $X_{0}$ are $k(\mathbf{G}, S)-2$ connected makes essential use of a result of Schulz Schu05] that analyzes connectivity properties of certain subsets of links in $X$.

We remark that unlike previous work on the positive direction for finiteness properties of arithmetic groups over function fields, our argument does not need assumptions on the geometry of the building $X$ neither with regard to type, nor degree of thickness, nor the dimensions of irreducible factors.

\subsection{Acknowledgements}

The first named author thanks Bernd Schulz for a thorough explaination of his PhD-thesis and Andrei Rapinchuck for helpful conversations.

\section{Reduction Theory}

Let $K$ be a global function field and suppose $\mathbf{G}$ is a connected, noncommutative, absolutely almost simple $K$-group. We assume that $\operatorname{rank}_{K}(\mathbf{G})=1$, we let $\mathbf{A} \leq \mathbf{G}$ be a maximal $K$-split torus, and we choose a minimal $K$-parabolic subgroup $\mathbf{P} \leq \mathbf{G}$ containing $\mathbf{A}$.

Note that the group of $K$-characters of $\mathbf{P}$ is infinite cyclic. Let $\chi$ be the generator for this group that is a positive multiple of the simple root associated with $\mathbf{P}$ in the root system of $\mathbf{G}$ with respect to $\mathbf{A}$.

Let $S$ be a finite, nonempty set of pairwise inequivalent valuations on $K$. Any $v \in S$ gives a norm $|\cdot|_{v}$ on $K$, and we let $K_{v}$ be the completion of $K$ with respect to this norm. For any $K$-group $\mathbf{H}$, we put $\mathbf{H}_{S}:=\prod_{v \in S} \mathbf{H}\left(K_{v}\right)$.

The statement of the next result below requires the definition of two sets. The first is the group

$$
\mathbf{P}_{S}^{0}:=\left\{\left.\left(g_{v}\right)_{v \in S} \in \mathbf{P}_{S}\left|\prod_{v \in S}\right| \chi\left(g_{v}\right)\right|_{v}=1\right\} .
$$

Second, for any $q>0$, we put:

$$
\mathbf{A}(q):=\left\{\left.\left(g_{v}\right)_{v \in S} \in \mathbf{A}_{S}\left|\prod_{v \in S}\right| \chi\left(g_{v}\right)\right|_{v} \geq q\right\} .
$$

We let $\mathcal{O}_{S}$ be the ring of $S$-integers in $K$ and we recall that $\mathbf{G}\left(\mathcal{O}_{S}\right)$ is a discrete subgroup of $\mathbf{G}_{S}$ via the diagonal embedding. The following theorem is a well known result from reduction theory. 
Theorem 2.1. There is a finite set of representatives $F \subset \mathbf{G}(K)$ for the double coset space $\mathbf{G}\left(\mathcal{O}_{S}\right) \backslash \mathbf{G}(K) / \mathbf{P}(K)$. Furthermore, for any such set $F$, there is some number $q>0$ and some compact set $C \subseteq \mathbf{G}_{S}$ such that

$$
\mathbf{G}_{S}=\mathbf{G}\left(\mathcal{O}_{S}\right) F \mathbf{P}_{S}^{0} \mathbf{A}(q) C .
$$

Proof. The finiteness of the double coset space $\mathbf{G}\left(\mathcal{O}_{S}\right) \backslash \mathbf{G}(K) / \mathbf{P}(K)$ is the statement of [Behr69, Satz 8]. Behr's proof needs a technical hypothesis (used for [Behr69, Satz 5]). However, Harder has removed the need for that hypothesis: [Hard69, Korollar 2.2.7] can be used as a replacement for [Behr69, Satz 5] in the proof.

The formula $\mathbf{G}_{S}=\mathbf{G}\left(\mathcal{O}_{S}\right) F \mathbf{P}_{S}^{0} \mathbf{A}(q) C$ follows from [Behr69, Satz 9]. Again, Behr uses a technical hypothesis, which has been subsequently removed. In this case, in addition to Harder's version of Behr's Satz 5, one needs to use a version of Behr's Satz 6 free from technical assumptions. This version is the main result of [Spri94]. Using these replacements, Behr's proofs apply.

We also remark that the discussion in [Hard69, page 52] implicitly contains a derivation of Theorem (2.1) in the context of Harder's version of reduction theory in positive characteristic.

q.e.d.

In the following section, $F, q$, and $C$ are fixed and satisfy Theorem 2.1.

\section{Horoballs}

We denote the Euclidean building corresponding to $\mathbf{G}\left(K_{v}\right)$ by $X_{v}$ and we let $X=\prod_{v \in S} X_{v}$. We also fix a vertex $e \in X$.

Let $\rho: \mathbb{R}_{\geq 0} \rightarrow X$ be the geodesic ray with $\rho(0)=e$ and such that $\rho(\infty)$ is the center of mass of the cell corresponding to $\mathbf{P}_{S}$ in the Tits boundary of $X$. Recall that this cell is the spherical join of the cells in the boundaries of the factors $X_{v}$ corresponding to $\mathbf{P}\left(K_{v}\right)$.

Let $\beta_{\rho}: X \rightarrow \mathbb{R}$ be the Busemann function induced by $\rho$ normalized so that $\beta(e)=0$. Sets of the form $\beta_{\rho}^{-1}\left(\mathbb{R}_{\geq t}\right)$ are called horoballs - or even horoballs based at $\mathbf{P}_{S}$ - in analogy with symmetric spaces. The next couple of lemmas explain how Theorem 2.1 naturally identifies a collection of horoballs that cover $X$. 
Lemma 3.1. For some $t \in \mathbb{R}$, we have

$$
\mathbf{P}_{S}^{0} \mathbf{A}(q) C \cdot e \subseteq \beta_{\rho}^{-1}\left(\mathbb{R}_{\geq t}\right) .
$$

Proof. Let $x \in \mathbf{P}_{S}^{0} \mathbf{A}(q) C \cdot e$. Note that $\beta_{\rho}(e)=0$ and since $C$ is compact, $C \cdot e \subseteq \beta_{\rho}^{-1}\left(\left[-t_{0}, t_{0}\right]\right)$ for some $t_{0}>0$. Furthermore, $\mathbf{P}_{S}^{0} \mathbf{A}(q) \subseteq \mathbf{P}_{S}$ fixes $\rho(\infty)$ and it follows from the definition of $\mathbf{P}_{S}^{0}$ and the fact that $\chi$ is a positive multiple of the simple root associated with $\mathbf{P}$ that $\mathbf{P}_{S}^{0}$ stabilizes horospheres based at $\mathbf{P}_{S}$, i.e., sets of the form $\beta_{\rho}^{-1}(r)$. Similarly, the definition of $\mathbf{A}(q)$ implies that there is a constant $L_{q} \geq 0$ such that for any $g \in \mathbf{A}(q)$, we have $g \cdot \beta_{\rho}^{-1}(r) \subseteq \beta_{\rho}^{-1}\left(\left[r-L_{q}, \infty\right)\right)$.

q.e.d.

Lemma 3.2. There is a $T \in \mathbb{R}$ such that

$$
\mathbf{G}\left(\mathcal{O}_{S}\right) F \cdot \beta_{\rho}^{-1}\left(\mathbb{R}_{\geq T}\right)=X .
$$

Proof. Combining Theorem 2.1] with Lemma 3.1 shows that

$$
\mathbf{G}_{S} \cdot e \subseteq \mathbf{G}\left(\mathcal{O}_{S}\right) F \cdot \beta_{\rho}^{-1}\left(\mathbb{R}_{\geq t}\right)
$$

for some $t \in \mathbb{R}$. The claim follows for some $T \leq t$ since any point in $X$ is a uniform bounded distance from a point in the orbit $\mathbf{G}_{S} \cdot e$.

q.e.d.

We have identified a cover of $X$ by horoballs. That is not a very interesting fact on its own, but we will use it to help us prove our ultimate goal in this section, which is to identify a pairwise disjoint collection of horoballs in $X$ with $\mathbf{G}\left(\mathcal{O}_{S}\right)$-invariant, cocompact complement. These pairwse disjoint horoballs will be retracts of the horoballs identified in Lemma 3.2, so we will want to know that any such horoball with its retract horoball removed has a precompact image in $\mathbf{G}\left(\mathcal{O}_{S}\right) \backslash X$. That is the goal of the corollary of the following:

Lemma 3.3. For $r \in \mathbb{R}$, any finite index subgroup of the discrete group $\mathbf{P}_{S}^{0} \cap \mathbf{G}\left(\mathcal{O}_{S}\right)$ acts cocompactly on the horosphere $\beta_{\rho}^{-1}(r)$.

Proof. $\mathbf{P}_{S}^{0}$ stabilizes the horosphere $\beta_{\rho}^{-1}(r)$ as in the proof of Lemma 3.1.

Let $y \in \beta_{\rho}^{-1}(r)$ and let $\Sigma_{v} \subseteq X_{v}$ be an apartment corresponding to a maximal $K_{v}$-split torus $\mathbf{A}_{v}$ in $\mathbf{P}$ containing $\mathbf{A}$. Let $\mathbf{R}^{\mathbf{u}}$ be the unipotent radical of $\mathbf{P}$. Recall that $\mathbf{R}^{\mathbf{u}}\left(K_{v}\right)$ acts transitively on the set of apartments 
in $X_{v}$ whose boundary sphere contains the chamber corresponding to $\mathbf{P}\left(K_{v}\right)$. Since $\mathbf{R}^{\mathbf{u}}\left(K_{v}\right) \leq \mathbf{P}_{S}^{0}$, there is some $g \in \mathbf{P}_{S}^{0}$ with $g \cdot y \in\left(\prod_{v \in S} \Sigma_{v}\right) \cap \beta_{\rho}^{-1}(r)$.

Note that $\left(\prod_{v \in S} \Sigma_{v}\right) \cap \beta_{\rho}^{-1}(r)$ is a codimension 1-subspace of $\prod_{v \in S} \Sigma_{v}$, and that $\left(\prod_{v \in S} \mathbf{A}_{v}\left(K_{v}\right)\right) \cap \mathbf{P}_{S}^{0}$ acts cocompactly on $\left(\prod_{v \in S} \Sigma_{v}\right) \cap \beta_{\rho}^{-1}(r)$. We have shown that $\mathbf{P}_{S}^{0}$ acts cocompactly on the horosphere $\beta_{\rho}^{-1}(r)$.

Now, we observe that $\mathbf{P}_{S}^{0} \cap \mathbf{G}\left(\mathcal{O}_{S}\right)$ is cocompact in $\mathbf{P}_{S}^{0}$ : the parabolic group $\mathbf{P}_{S}^{0}$ decomposes as a product $\mathbf{R}_{S}^{\mathbf{u}} \mathbf{L}_{S} \mathbf{A}_{S}^{0}$ where $\mathbf{L}$ is a reductive group of $K$-rank 0. Note that the subgroup of $S$-integer points in $\mathbf{R}_{S}^{\mathbf{u}}$ is cocompact since $\mathbf{R}^{\mathbf{u}}$ is unipotent; for the subgroup of $S$-integer points in $\mathbf{L}_{S}$, cocompactness follows from [Hard69, Korollar 2.2.7]; and for $\mathbf{A}_{S}^{0}$, cocompactness of the $S$-integer subgroup follows from Dirichlet's unit theorem. Therefore, $\mathbf{P}_{S}^{0} \cap \mathbf{G}\left(\mathcal{O}_{S}\right)$ is cocompact in $\mathbf{P}_{S}^{0}$ and thus so is any finite index subgroup of $\mathbf{P}_{S}^{0} \cap \mathbf{G}\left(\mathcal{O}_{S}\right)$. The claim now follows.

q.e.d.

Corollary 3.4. For any $f \in F$ and any $R \geq 0$, the quotient

$$
\mathbf{G}\left(\mathcal{O}_{S}\right) \backslash^{f \cdot \beta_{\rho}^{-1}([-R, R])} \subseteq \mathbf{G}\left(\mathcal{O}_{S}\right) \backslash X
$$

is compact.

Proof. For $f=1$, the claim is immediate from Lemma 3.3, If $f \neq 1$, we replace the role of $\mathbf{P}_{S}^{0} \cap \mathbf{G}\left(\mathcal{O}_{S}\right)=\mathbf{P}\left(\mathcal{O}_{S}\right)$ in Lemma 3.3 with $f \mathbf{P}\left(\mathcal{O}_{S}\right) f^{-1} \cap$ $\mathbf{G}\left(\mathcal{O}_{S}\right)$. This is a finite index subgroup of $f \mathbf{P}\left(\mathcal{O}_{S}\right) f^{-1}$ since $f \in \mathbf{G}(K)$, see e.g. [Marg91, Lemma 3.1.1(iv)].

q.e.d.

We let $\beta_{f \cdot \rho}: X \rightarrow \mathbb{R}$ be the Busemann function for the geodesic ray $f \cdot \rho$. Thus, $f \cdot \beta_{\rho}^{-1}(r)=\beta_{f \cdot \rho}^{-1}(r)$.

Lemma 3.5. There is some $R_{f}>0$ such that $\mathbf{G}\left(\mathcal{O}_{S}\right) \cdot e \cap \beta_{f \cdot \rho}^{-1}\left(\left[R_{f}, \infty\right)\right)=\emptyset$.

Proof. Let $\Gamma_{f}=f \mathbf{P}\left(\mathcal{O}_{S}\right) f^{-1} \cap \mathbf{G}\left(\mathcal{O}_{S}\right)$ and let $M_{r}$ be the Hausdorff distance between the orbit $\Gamma_{f} f \cdot \rho(r)$ and the horosphere $\beta_{f \cdot \rho}^{-1}(r)$. Note that $M_{r_{2}} \leq M_{r_{1}}$ when $r_{1}<r_{2}$.

Let $a \in \mathbf{A}_{S}$ be defined by $a=\left(a_{v}\right)_{v \in S}$ where $a_{v} \in \mathbf{A}\left(K_{v}\right)$ is such that $\left|\chi\left(a_{v}\right)\right|_{v}>1$, and let $a_{f}=f a f^{-1}$. Since $f a_{v} f^{-1}$ acts by translations on $f \cdot \Sigma_{v}$, we have $\beta_{f \cdot \rho}\left(a_{f}^{n} \cdot e\right)=n L+\beta_{f \cdot \rho}(e)$ for some $L>0$. Note that for any $u \in f \mathbf{R}^{\mathrm{u}}\left(\mathcal{O}_{S}\right) f^{-1} \cap \mathbf{G}\left(\mathcal{O}_{S}\right)$, the sequence $a_{f}^{-n} u a_{f}^{n}$ converges to 1 in $\mathbf{G}_{S}$. By Ragh72, Theorem I.1.12], the sequence $\mathbf{G}\left(\mathcal{O}_{S}\right) a_{f}^{-n} u a_{f}^{n}$ contains no 
convergent subsequence in $\mathbf{G}\left(\mathcal{O}_{S}\right) \backslash \mathbf{G}_{S}$. It follows that there is some $N$ such that $d\left(a_{f}^{n} \cdot e, \mathbf{G}\left(\mathcal{O}_{S}\right) \cdot e\right)>L+M_{0}$ for all $n \geq N$.

Let $R_{f}=\max \left(N L, \beta_{f \cdot \rho}(e)\right)$ and suppose $x \in \beta_{f \cdot \rho}^{-1}\left(\left[R_{f}, \infty\right)\right)$. We claim that $x \notin \mathbf{G}\left(\mathcal{O}_{S}\right) \cdot e$. Indeed, we can choose $n \geq N$ such that

$$
\left|\beta_{f \cdot \rho}\left(a_{f}^{n} \cdot e\right)-\beta_{f \cdot \rho}(x)\right|<L
$$

so we can choose $\gamma \in \Gamma_{f}$ such that

$$
d\left(a_{f}^{n} \cdot e, \gamma \cdot x\right) \leq L+M_{0}
$$

Thus $\gamma \cdot x \notin \mathbf{G}\left(\mathcal{O}_{S}\right) \cdot e$ and so $x \notin \mathbf{G}\left(\mathcal{O}_{S}\right) \cdot e$.

q.e.d.

We let $d>0$ be the maximum of the distances from points in the horosphere $\beta_{f \cdot \rho}^{-1}\left(R_{f}\right)$ to the orbit $\mathbf{G}\left(\mathcal{O}_{S}\right) \cdot e$ as $f$ ranges through $F$. Note that $d$ is finite by Lemma 3.3 .

Lemma 3.6. Let $\gamma \in \mathbf{G}\left(\mathcal{O}_{S}\right)$ and suppose

$$
\gamma \cdot \beta_{f \cdot \rho}^{-1}\left(\left[R_{f}+d, \infty\right)\right) \cap \beta_{\hat{f} \cdot \rho}^{-1}\left(\left[R_{\hat{f}}+d, \infty\right)\right) \neq \emptyset .
$$

Then $\gamma \in\left(f \mathbf{P} f^{-1}\right)\left(\mathcal{O}_{S}\right)$, and $f=\hat{f}$, and

$$
\gamma \cdot \beta_{f \cdot \rho}^{-1}\left(\left[R_{f}+d, \infty\right)\right)=\beta_{\hat{f} \cdot \rho}^{-1}\left(\left[R_{\hat{f}}+d, \infty\right)\right) .
$$

Proof. Let $\Sigma \subseteq X$ be an apartment whose boundary sphere at infinity contains the cells corresponding to $\gamma f \mathbf{P}_{S} f^{-1} \gamma^{-1}$ and $\hat{f} \mathbf{P}_{S} \hat{f}^{-1}$. If $\gamma f \mathbf{P}_{S} f^{-1} \gamma^{-1} \neq$ $\hat{f} \mathbf{P}_{S} \hat{f}^{-1}$ then $\operatorname{rank}_{K}(\mathbf{G})=1$ implies that these are opposite cells at infinity, and thus the triple intersection

$$
\Sigma \cap \gamma \cdot \beta_{f \cdot \rho}^{-1}\left(\left[R_{f}+d, \infty\right)\right) \cap \beta_{\hat{f} \cdot \rho}^{-1}\left(\left[R_{\hat{f}}+d, \infty\right)\right)
$$

is contained in a metric neighborhood of a hyperplane in $\Sigma$.

We choose

$$
x \in \Sigma \cap \gamma \cdot \beta_{f \cdot \rho}^{-1}\left(\left[R_{f}+d, \infty\right)\right) \cap \beta_{\hat{f} \cdot \rho}^{-1}\left(R_{\hat{f}}\right) .
$$

It follows from the choice of $d$ that there is some $y \in \mathbf{G}\left(\mathcal{O}_{S}\right) \cdot e$ such that $d(x, y) \leq d$. Therefore, $\beta_{\gamma f \cdot \rho}(y) \geq R_{f}$ which contradicts Lemma 3.5. Hence $\gamma f \mathbf{P}_{S} f^{-1} \gamma^{-1}=\hat{f} \mathbf{P}_{S} \hat{f}^{-1}$ which is to say that $f=\hat{f}$ and $\gamma \in\left(f \mathbf{P}_{S} f^{-1}\right)\left(\mathcal{O}_{S}\right)$.

Furthermore, $\gamma$ preserves distances from $\mathbf{G}\left(\mathcal{O}_{S}\right) \cdot e$, so the result follows.

q.e.d. 
Let $\mathcal{H}=\mathbf{G}\left(\mathcal{O}_{S}\right) \cdot\left\{\beta_{f \cdot \rho}^{-1}\left(\left(R_{f}+d, \infty\right)\right) \mid f \in F\right\}$. This is a collection of open horoballs.

Theorem 3.7. $\mathcal{H}$ is a collection of pairwise disjoint horoballs; $X \backslash \mathcal{H}$ is $\mathrm{G}\left(\mathcal{O}_{S}\right)$-invariant and cocompact.

Proof. The result follows from the definition of $\mathcal{H}$, Lemma 3.6, and Corollary 3.4

q.e.d.

\section{Connectivity of Horospheres in General Position}

Let $\Delta$ be a spherical building. We consider $\Delta$ as a metric space with the angular metric $d$, i.e., every apartment is a unit sphere. For any point $x \in \Delta$, we define the closed hemisphere complex $\Delta^{\geq \frac{\pi}{2}}(x)$ to be the subcomplex spanned by all vertices in the set $\left\{y \in \Delta \mid d(y, x) \geq \frac{\pi}{2}\right\}$, the open hemisphere complex $\Delta^{>\frac{\pi}{2}}(x)$ as the subcomplex spanned by all vertices in the set $\left\{y \in \Delta \mid d(y, x)>\frac{\pi}{2}\right\}$, and we define the the equator as the set of

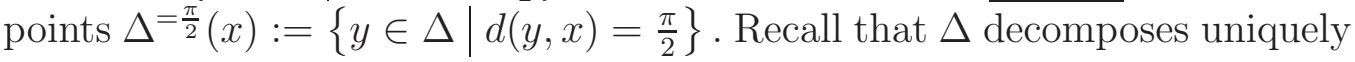
as the spherical join of irreducible factors

$$
\Delta=\Delta_{1} * \cdots * \Delta_{k}
$$

where the decomposition is determined by the geometry of chambers as follows:

Lemma 4.1. In an irreducible spherical building, every edge has angular length strictly less than $\frac{\pi}{2}$. Consequently, in any spherical building, an edge has angular length $\frac{\pi}{2}$ if and only if it joins two vertices from different irreducible factors.

Proof. We start with the following observation from spherical geometry: suppose all edges and angles in a spherical triangle are at most $\frac{\pi}{2}$; if one edge has length exactly $\frac{\pi}{2}$ then so has at least one of the other edges (in fact, also at least two of the angles will be right angles).

We apply this observation to the vertices of a chamber $C$. Let $v_{1}, v_{2}, \ldots, v_{k}$ be a maximal collection of vertices that have pairwise distance

$\frac{\pi}{2}$. Then every other vertex has distance strictly less than $\frac{\pi}{2}$ to at least one 
of them (by maximality of the collection) and therefore to exactly one of them (by the observation). It follows that being of distance strictly less than $\frac{\pi}{2}$ is an equivalence relation on the set of vertices of $C$ with $k$ equivalence classes. This defines a decomposition of the underlying Coxeter complex as a spherical join and induces a decomposition of the building into irreducible factors.

q.e.d.

Let $\Delta_{\text {hor }}(x)$ be the join of all irreducible factors completely contained in the equator, and let $\Delta_{\text {ver }}(x)$ be the join of the other factors. Clearly $\Delta=\Delta_{\text {hor }}(x) * \Delta_{\text {ver }}(x)$.

Lemma 4.2. Let $C \subseteq \Delta$ be a chamber. Then for any equatorial simplex $\tau \subseteq \Delta^{=\frac{\pi}{2}}(x)$ contained in $C$, the following are equivalent:

1. We have $\tau \subseteq \Delta_{\text {hor }}$.

2. The simplex $\tau$ has Hausdorff distance $\frac{\pi}{2}$ from any non-equatorial vertex in $\Delta$.

3. The simplex $\tau$ has Hausdorff distance $\frac{\pi}{2}$ from any non-equatorial vertex in $C$.

Proof. The implications (11) $\Rightarrow(21)$ and (21) $\Rightarrow(3)$ are obvious. It remains to show that (3) implies (11).

We will show that $v \notin \Delta_{\text {ver }}$ for each vertex $v \in \tau$. This implies that all vertices of $\tau$ belong to $\Delta_{\text {hor }}$ and thus proves the claim.

Let $v$ be a vertex of $\tau$ and let $\Delta_{i}$ be a vertical irreducible factor of $\Delta$. Note that a chamber in $\Delta_{i}$ cannot have all its vertices in the equator. It follows that $C \cap \Delta_{i}$ contains a non-equatorial vertex. Since this vertex is connected to $v$ by an edge of length $\frac{\pi}{2}$, it follows from Fact (4.1) that $v \notin \Delta_{i}$. Since the same argument proves that $v$ is not in any irreducible factor of $\Delta_{\text {ver }}$, we have $v \notin \Delta_{\text {ver }}$.

q.e.d.

Connectivity properties of hemisphere complexes are given by the following:

Theorem 4.3 (Bernd Schulz, Schu05]). Assume that $\Delta$ is a thick spherical building. The closed hemisphere complex $\Delta \geq \frac{\pi}{2}(x)$ is $(\operatorname{dim}(\Delta)-1)$ connected.

The open hemisphere complex $\Delta^{>\frac{\pi}{2}}(x)$ is $\left(\operatorname{dim}\left(\Delta_{\mathrm{ver}}(x)\right)-1\right)$-connected. 
As a first application, we shall deduce the connectivity of horospheres "in general position".

Proposition 4.4. Let $X$ be a thick Euclidean building and let

$$
\beta: X \longrightarrow \mathbb{R}
$$

be a Busemann function that is non-constant on each edge of $X$. Then, any horosphere $\beta^{-1}(r)$ is $(\operatorname{dim}(X)-2)$-connected.

Before we embark on the proof, we need to state a version of the MorseLemma that fuels Bestvina-Brady type combinatorial Morse-theory as introduced in [BeBr97. Let $X$ be a piecewise Euclidean complex and let $h: X \rightarrow \mathbb{R}$ be a function that is affine on cells and non-constant on edges. The descending link $\operatorname{Lk}^{\downarrow}(v)$ of a vertex $v \in X$ is the subcomplex of $\operatorname{Lk}(v)$ defined by all cells $c$ in $X$ containing $v$ as the point where $h$ attains its maximum on $c$.

Morse Lemma 4.5. Let $X$ and $h$ be as above, and let $r<s$ be real numbers chosen such that the preimage $h^{-1}([r, s])$ does not contain a complete edge. Then the sublevel set $h^{-1}((-\infty, s])$ is homotopy equivalent to the sublevel set $h^{-1}((-\infty, r])$ with descending links of vertices in $h^{-1}((r, s])$ conned off.

We shall not give a proof of the Morse-Lemma here since we will prove a slightly more general version later. We just remark that the version above is essentially the Morse-Lemma from [BuGo99, Lemma 7].

Observation 4.6. Let $C_{\infty}$ be a chamber of the spherical building at infinity that contains the end $e_{\infty}$. Note that $X$ is covered by apartments containing $C_{\infty}$ and that $\beta$ is affine on all those apartments. In each such apartment, there are only finitely many edges up to translation. Moreover, any two such apartments have a common sector representing $C_{\infty}$. Thus, there are only finitely many "parallelism classes" of edges in $X$.

q.e.d.

Proof of Proposition 4.4. Let $v \in X$ be a vertex. The link of $v$ is a spherical building $\Delta:=\operatorname{Lk}(v)$. There is a unique geodesic ray issuing from $v$ toward $e_{\infty}$. This geodesic represents the gradient $\nabla \beta \in \Delta=\operatorname{Lk}(v)$ of the Busemann function defined by $e_{\infty}$. Observe that directions issuing from $v$ are descending if they span an obtuse angle with the gradient. It follows that the descending link $\operatorname{Lk}^{\downarrow}(v)$ is the open hemisphere complex $\Delta^{>\frac{\pi}{2}}(\nabla \beta)$. 
Since there are no horizontal edges, the open hemisphere complex and the closed hemisphere complex coincide. It follows that descending links are $(\operatorname{dim}(X)-2)$-connected.

It follows form Observation (4.6) that there is a constant $\varepsilon>0$ so that for any two vertices $v, w \in X$ joined by an edge, we have $|\beta(v)-\beta(w)|>\varepsilon$.

Our choice of $\varepsilon$ ensures that no preimage $\beta^{-1}([s, s+\varepsilon])$ contains a complete edge. Thus, the Morse-Lemma implies that, for any $s \in \mathbb{R}$, $\beta^{-1}((-\infty, s+\varepsilon])$ is homotopy equivalent to $\beta^{-1}((-\infty, s])$ with descending links conned off. As descending links are $(\operatorname{dim}(X)-2)$-connected, we find that the inclusion

$$
\beta^{-1}((-\infty, s]) \hookrightarrow \beta^{-1}((-\infty, s+\varepsilon])
$$

induces isomorphisms in $\pi_{n}$ for $n \leq \operatorname{dim}(X)-2$. Iterating, we obtain that for any $s>r$ the inclusion

$$
\beta^{-1}((-\infty, r]) \hookrightarrow \beta^{-1}((-\infty, r+\varepsilon]) \hookrightarrow \beta^{-1}((-\infty, r+2 \varepsilon]) \hookrightarrow \cdots \hookrightarrow X
$$

induces isomorphisms in $\pi_{n}$ for $n \leq \operatorname{dim}(X)-2$. Since $X$ is contractible, it follows that sublevel sets are $(\operatorname{dim}(X)-2)$-connected.

q.e.d.

\section{Toward a Secondary Morse Function}

Ultimately, we want to deal with horospheres that are not necessarily in general position, i.e., the corresponding Busemann function might be constant on some edges. To overcome this obstacle, we construct a secondary Morse function that will allow us to break ties.

Let $X$ be an irreducible Euclidean building. The link of any simplex $\tau$, is the union of all those simplices $\sigma$ disjoint from $\tau$ such that $\sigma \cup \tau$ is a simplex. This link, $\operatorname{Lk}(\tau)$ is a spherical building, and we may alternatively think of its points as directions issuing from the barycenter $\stackrel{\circ}{\tau}$ of $\tau$ that are perpendicular to $\tau$ - one way to make sense of perpendicularity is to recall that $X$ is a CAT(0) space and that $\tau$ is a convex subset. We note that this way the link $\operatorname{Lk}(\tau)$ is endowed with an angular metric so that each apartment in $\operatorname{Lk}(\tau)$ is a unit sphere.

Let $\beta: X \longrightarrow \mathbb{R}$ be a Busemann function on $X$ corresponding to a point $e_{\infty}$ at infinity. We call a simplex $\tau \subseteq X$ horizontal if $\beta$ restricts to a constant map on $\tau$. For a horizontal simplex $\tau$, the unique geodesic ray from 
the barycenter $\stackrel{\circ}{\tau}$ to the end $e_{\infty}$ is perpendicular to $\tau$ and thus determines a direction $\nabla \beta \in \operatorname{Lk}(\tau)$, to which we refer as the gradient of $\beta$.

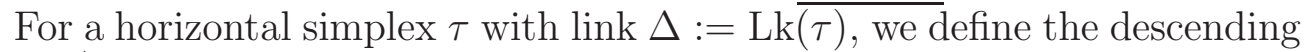
link $\operatorname{Lk}_{\beta}^{\downarrow}(\tau)$ as the subcomplex defined by those simplices in $X$ that contain $\tau$ as a face and where $\beta$ is maximal exactly along the face $\tau$. It is obvious that $\operatorname{Lk}_{\beta}^{\downarrow}(\tau)$ coincides with the open hemisphere complex $\Delta^{>\frac{\pi}{2}}(\nabla \beta)$. We define the equatorial link $\operatorname{Lk}_{\beta}=(\tau):=\Delta^{=\frac{\pi}{2}}(\nabla \beta)$, i.e., as the set of directions along which $\beta$ does not change. We also define the vertical link as $\operatorname{Lk}_{\mathrm{ver}}(\tau):=\Delta_{\text {ver }}$ and the horizontal link as $\operatorname{Lk}_{\text {hor }}(\tau):=\Delta_{\text {hor }}$.

Lemma 5.1. Let $\tau$ be a simplex in $X$ and let $\sigma_{1}, \sigma_{2}$ be two simplices in $\operatorname{Lk}(\tau)$ that span a simplex $\sigma_{1} \cup \sigma_{2}$. Then the following are equivalent:

1. The simplices $\sigma_{1}$ and $\sigma_{2}$ have distance $\frac{\pi}{2}$ in $\operatorname{Lk}(\tau)$.

2. There is a decomposition $\operatorname{Lk}(\tau)=\Delta_{1} * \Delta_{2}$ of the link as a spherical join so that $\sigma_{1} \subseteq \Delta_{1}$ and $\sigma_{2} \subseteq \Delta_{2}$.

3. The orthogonal projection $\operatorname{proj}_{\tau \cup \sigma_{1}}\left(\sigma_{2}\right)$ is contained in $\tau$. (The orthogonal projection can be carried out in any Euclidean apartment containing $\sigma_{1}, \sigma_{2}$, and $\tau$. The result is independent of which apartment was chosen.)

\section{Proof.}

(11) $\Longrightarrow(2)$ This follows from Lemma (4.1).

$(2) \Longrightarrow(3)$ clear.

(3) $\Longrightarrow(1)$ clear.

Lemma 5.2. For any horizontal simplex $\tau$, there is a unique face $\tau^{\min } \leq \tau$ such that for any proper face $\sigma<\tau$, we have the equivalence

$$
\tau \backslash \sigma \in \operatorname{Lk}_{\text {hor }}(\sigma) \quad \Longleftrightarrow \quad \tau^{\min } \leq \sigma<\tau
$$

More precisely, using any chamber $C$ containing $\tau$, the face $\tau^{\min }$ can be described as the smallest face of $\tau$ containing the set

$$
\left\{\operatorname{proj}_{\tau}(v) \mid v \text { vertex in } C, \beta(v) \neq \beta(\tau)\right\} \text {. }
$$


Proof. Note that uniqueness of $\tau^{\text {min }}$ is obvious. It remains to show that for any choice of the chamber $C$ the face $\tau^{\text {min }}$ defined above satisfies

$$
\tau \backslash \sigma \in \operatorname{Lk}_{\text {hor }}(\sigma) \Longleftrightarrow \tau^{\min } \leq \sigma<\tau
$$

for each proper face $\sigma<\tau$.

By Lemma (4.2), we have $\tau \backslash \sigma \subseteq \operatorname{Lk}_{\text {hor }}(\sigma)$ if and only if every nonequatorial vertex $v \in C$ has distance $\frac{\pi}{2}$ to $\tau \backslash \sigma$, which by Lemma (5.1) happens if and only if $\operatorname{proj}_{\mathcal{\tau}}(v) \in \sigma$. This, in turn, is equivalent to $\tau^{\text {min }} \leq \sigma$ by construction of $\tau^{\text {min }}$.

q.e.d.

Let $\sigma$ be a face of $\tau$. Note that in any Euclidean apartment containing $\tau$, the orthogonal projection onto the affine subspace spanned by $\sigma$ factors through the orthogonal projection onto the subspace spanned by $\tau$. It is now easy to make the following:

Observation 5.3. Suppose $\tau^{\text {min }} \leq \sigma \leq \tau$ for some horizontal simplices. Then $\tau^{\mathrm{min}}=\sigma^{\mathrm{min}}$. In particular, $\tau^{\mathrm{min}}=\left(\tau^{\mathrm{min}}\right)^{\mathrm{min}}$.

Proof. Let $C$ be a chamber containing $\tau$. Then, for any vertex $v \in C$ not on the level of $\tau$, we have

$$
\operatorname{proj}_{\sigma}(v)=\operatorname{proj}_{\sigma}\left(\operatorname{proj}_{\tau}(v)\right)=\operatorname{proj}_{\tau}(v)
$$

since $\operatorname{proj}_{\tau}(v) \in \tau^{\text {min }} \subseteq \sigma$ by hypothesis.

q.e.d.

We now define two relations on horizontal simplices. We define going up as

$$
\sigma \nearrow \tau \quad: \Longleftrightarrow \sigma=\tau^{\min } \neq \tau
$$

and going down as

$$
\tau \searrow \sigma \quad \Longleftrightarrow \quad \tau^{\min } \not \leq \sigma<\tau \text {. }
$$

We define a move as either going up or going down and write $\tau_{1} \rightarrow \tau_{2}$ if there is a move from $\tau_{1}$ to $\tau_{2}$. The main result of this section is the following

Proposition 5.4. There is a uniform bound, depending only on the dimension of $X$, on the length of any sequence of moves. 
Thus, we can define the depth $\operatorname{dp}(\tau)$ of a simplex as the length of a longest sequence of moves starting at $\tau$. Assuming for a moment that the depth is well defined, we have the following:

Observation 5.5. If there is a move from $\tau_{1}$ to $\tau_{2}$, then $\operatorname{dp}\left(\tau_{1}\right)>\operatorname{dp}\left(\tau_{2}\right)$ since we can put the move from $\tau_{1}$ to $\tau_{2}$ in front of a sequence starting at $\tau_{2}$ and obtain a longer chain starting at $\tau_{1}$.

q.e.d.

The remainder of this section is entirely devoted to the proof of Proposition (5.4) and independent of the other parts of the paper.

Let us begin by collecting some elementary properties of the two types of moves. We begin with transitivity.

Lemma 5.6. It never happens that $\tau_{1} \nearrow \tau_{2} \nearrow \tau_{3}$. In particular, the symmetric closure of $\nearrow$ is transitive for silly reasons.

Proof. Suppose $\tau_{1} \nearrow \tau_{2} \nearrow \tau_{3}$. Then, by Observation (5.3), $\tau_{1}=\tau_{2}^{\min }=$ $\left(\tau_{3}^{\mathrm{min}}\right)^{\mathrm{min}}=\tau_{3}^{\mathrm{min}}=\tau_{2}$ contradicting $\tau_{1}<\tau_{2}$.

q.e.d.

Lemma 5.7. The relation $\searrow$ is transitive.

Proof. Suppose $\tau_{1} \searrow \tau_{2} \searrow \tau_{3}$. Then $\tau_{1}^{\min } \not \leq \tau_{2}<\tau_{1}$ and $\tau_{2}^{\min } \not \leq \tau_{3}<\tau_{2}$. It follows immediately that $\tau_{3}<\tau_{1}$. Also, $\tau_{1}^{\min } \not \leq \tau_{2}$ and $\tau_{3} \leq \tau_{2}$ imply that $\tau_{1}^{\min } \not \leq \tau_{3}$. Thus, $\tau_{1} \searrow \tau_{3}$.

q.e.d.

The next batch of lemmata deals with chains of simplices

$$
\sigma_{1} \nearrow \tau_{1} \searrow \sigma_{2} \nearrow \tau_{2} \searrow \cdots
$$

alternatingly going up and down.

Lemma 5.8. If some horizontal simplices satisfy

$$
\sigma_{1} \nearrow \tau_{1} \searrow \sigma_{2}
$$

then we have

$$
\sigma_{1}=\left(\sigma_{1} \cup \sigma_{2}\right)^{\min } \text { and } \sigma_{1} \cup \sigma_{2} \searrow \sigma_{2} .
$$

In particular, we have $\sigma_{1} \nearrow \sigma_{1} \cup \sigma_{2} \searrow \sigma_{2}$ unless $\sigma_{1} \searrow \sigma_{2}$.

Proof. From Observation [5.3, we deduce $\sigma_{1}=\left(\sigma_{1} \cup \sigma_{2}\right)^{\mathrm{min}}$. On the other hand, $\sigma_{1}=\tau_{1}^{\min } \not \leq \sigma_{2}$, whence $\sigma_{2}<\sigma_{1} \cup \sigma_{2}$ and $\sigma_{1} \cup \sigma_{2} \searrow \sigma_{2}$. $\quad$ q.e.d. 
Lemma 5.9. Let $\sigma_{1}$ and $\sigma_{2}$ be two simplices whose union is a horizontal simplex. Let $v \in \operatorname{Lk}\left(\sigma_{1} \cup \sigma_{2}\right)$ be a vertex, and let $\sigma \subseteq \sigma_{1} \cup \sigma_{2}$ be the carrier of $\operatorname{proj}_{\sigma_{1} \cup \sigma_{2}}(v)$, i.e., the smallest face of $\sigma_{1} \cup \sigma_{2}$ containing $\operatorname{proj}_{\sigma_{1} \cup \sigma_{2}}(v)$. Then $\operatorname{proj}_{\sigma_{2} \cup\{w\}}(v) \notin \sigma_{2}$ for every vertex $w \in \sigma \backslash \sigma_{2}$. In particular, $v$ and $w$ are in the same irreducible factor of $\operatorname{Lk}\left(\sigma_{2}\right)$.

Proof. The point $\operatorname{proj}_{\sigma_{2} \cup \sigma_{1}}(v)$ is a convex combination of the vertices in $\sigma_{2} \cup \sigma_{1}$. Since $w$ lies in the carrier of $\operatorname{proj}_{\sigma_{2} \cup \sigma_{1}}(v)$, we can infer that the $w$ coordinate of $\operatorname{proj}_{\sigma_{2} \cup \sigma_{1}}(v)$ is non-zero. From $\operatorname{proj}_{\sigma_{2} \cup\{w\}}(w)=w$ we can now deduce that $\operatorname{proj}_{\sigma_{2} \cup\{w\}}(v)=\operatorname{proj}_{\sigma_{2} \cup\{w\}}\left(\operatorname{proj}_{\sigma_{2} \cup \sigma_{1}}(v)\right)$ still has a non-zero $w$ coordinate, whence it cannot lie in $\sigma_{2}$.

q.e.d.

Lemma 5.10. Let $\sigma_{1}$ and $\sigma_{2}$ be two simplices whose union is a horizontal simplex. Suppose $\sigma_{1}=\left(\sigma_{1} \cup \sigma_{2}\right)^{\mathrm{min}}$. Then any vertex $w \in \sigma_{1} \backslash \sigma_{2}$ lies in $\operatorname{Lk}_{\mathrm{ver}}\left(\sigma_{2}\right)$.

Proof. Note that $w \in \sigma_{1}=\left(\sigma_{1} \cup \sigma_{2}\right)^{\text {min }}$. It follows that there is a vertex $v \in \operatorname{Lk}\left(\sigma_{1} \cup \sigma_{2}\right)$ with $\beta(v) \neq \beta\left(\sigma_{1} \cup \sigma_{2}\right)$ that is a witness for $w \in \sigma_{1}=$ $\left(\sigma_{1} \cup \sigma_{2}\right)^{\mathrm{min}}$, i.e., $w$ belongs to the smallest simplex containing $\operatorname{proj}_{\sigma_{1} \cup \sigma_{2}}(v)$. It then follows from Lemma (5.9) that $w$ belongs to the same irreducible factor of $\operatorname{Lk}\left(\sigma_{2}\right)$ as $v$. As $v \in \operatorname{Lk}_{\mathrm{ver}}\left(\sigma_{2}\right)$, we have $w \in \operatorname{Lk}_{\mathrm{ver}}\left(\sigma_{2}\right)$. $\quad$ q.e.d.

Corollary 5.11. Let

$$
\sigma_{1} \nearrow \tau_{1} \searrow \sigma_{2} \nearrow \tau_{2}
$$

be horizontal simplices. Then $\tau_{2} \cup \sigma_{1}$ is a horizontal simplex.

Proof. Note that $\sigma_{1} \cup \sigma_{2}$ is a horizontal simplex and $\left(\sigma_{1} \cup \sigma_{2}\right) \backslash \sigma_{2}$ is a simplex in the vertical link of $\sigma_{2}$ by Lemma (5.10).

On the other hand, $\tau_{2} \backslash \sigma_{2}$ is a simplex in the horizontal link of $\sigma_{2}$ as $\sigma_{2} \nearrow \tau_{2}$. It follows that $\left(\sigma_{1} \cup \sigma_{2}\right) \backslash \sigma_{2}$ and $\tau_{2} \backslash \sigma_{2}$ span a simplex in $\operatorname{Lk}\left(\sigma_{2}\right)$. The claim follows.

q.e.d.

Now, we are ready to discuss shortening of alternating chains and to rule out the existence of cycles. We start by ruling out cycles of length 2 .

Observation 5.12. There do not exist horizontal simplices $\sigma$ and $\tau$ with $\sigma \nearrow \tau$ and $\tau \searrow \sigma$ since $\sigma \nearrow \tau$ implies $\sigma=\tau^{\text {min }}$ whereas $\tau \searrow \sigma$ implies $\tau^{\min } \not \leq \sigma$.

q.e.d. 
Lemma 5.13. Given an alternating chain

$$
\sigma_{1} \nearrow \tau_{1} \searrow \sigma_{2} \nearrow \tau_{2}
$$

we have $\left(\sigma_{1} \cup \tau_{2}\right)^{\min }=\sigma_{1}$.

Proof. Let $v \in \operatorname{Lk}\left(\sigma_{1} \cup \tau_{2}\right)$ with $\beta(v) \neq \beta\left(\sigma_{1} \cup \tau_{2}\right)$. Since $\sigma_{2} \nearrow \tau_{2}$, we have $\sigma_{1} \cup \sigma_{2} \subseteq \sigma_{1} \cup \tau_{2}$; also, $\left(\sigma_{1} \cup \sigma_{2}\right)^{\text {min }}=\sigma_{1}$ by Lemma (5.8). Therefore $\operatorname{proj}_{\sigma_{1} \cup \sigma_{2}}(v) \in \sigma_{1}$. Also note that $\operatorname{proj}_{\tau_{2}}(v) \in \sigma_{2}$ as $\sigma_{2}=$ $\tau_{2}^{\min }$. Hence, $\operatorname{proj}_{\sigma_{1} \cup \tau_{2}}(v) \in \sigma_{1} \cup \sigma_{2}$. We conclude that $\operatorname{proj}_{\sigma_{1} \cup \tau_{2}}(v)=$ $\operatorname{proj}_{\sigma_{1} \cup \sigma_{2}}\left(\operatorname{proj}_{\sigma_{1} \cup \tau_{2}}(v)\right)=\operatorname{proj}_{\sigma_{1} \cup \sigma_{2}}(v) \in \sigma_{1}$. Thus, by Lemma (5.2),

$$
\left(\sigma_{1} \cup \tau_{2}\right)^{\min } \leq \sigma_{1} \leq \sigma_{1} \cup \sigma_{2} \leq \sigma_{1} \cup \tau_{2},
$$

whence

$$
\left(\sigma_{1} \cup \tau_{2}\right)^{\min }=\left(\sigma_{1} \cup \sigma_{2}\right)^{\min }=\sigma_{1}
$$

by Observation (5.3) and Lemma (5.8).

q.e.d.

Shortening Lemma 5.14. Any alternating chain

$$
\sigma_{1} \nearrow \tau_{1} \searrow \sigma_{2} \nearrow \tau_{2}
$$

can be shortened to

$$
\sigma_{1} \nearrow\left(\sigma_{1} \cup \tau_{2}\right) \searrow \tau_{2} \text { or } \sigma_{1} \nearrow \tau_{1} \searrow \tau_{2}
$$

In the second case, one actually has a shorter chain $\sigma_{1} \searrow \tau_{2}$. For technical reasons, however, it is more convenient to keep the chain going up initially.

Proof. By Lemma (5.13), we have $\sigma_{1}=\left(\sigma_{1} \cup \tau_{2}\right)^{\mathrm{min}}$. Also, by Observation (5.12), we have $\sigma_{1} \neq \sigma_{2}=\tau_{2}^{\text {min }}$ whence $\tau_{2} \neq \sigma_{1} \cup \tau_{2}$. It follows that $\sigma_{1} \cup \tau_{2} \searrow \tau_{2}$.

If $\sigma_{1} \neq \sigma_{1} \cup \tau_{2}$, we find $\sigma_{1} \nearrow\left(\sigma_{1} \cup \tau_{2}\right) \searrow \tau_{2}$.

If $\sigma_{1}=\sigma_{1} \cup \tau_{2}$, we find $\tau_{2}<\sigma_{1}<\tau_{1}$ and therefore $\tau_{1} \searrow \tau_{2} . \quad$ q.e.d.

As a consequence, we can rule out cycles of arbitrary length.

Corollary 5.15. No sequence of moves enters a cycle. 
Proof. Since $\nearrow$ and $\searrow$ are both transitive (Lemmata (5.6) and (5.7)), any minimum length cycle has to alternate between $\nearrow$ and $\searrow$. By the Shortening Lemma (5.14), a minimum length cycle can go up at most once. Thus, a minimum length cycle is alternating of length two. This, however, is ruled out by Observation (5.12).

q.e.d.

Lemma 5.16. Let

$$
\sigma_{1} \nearrow \tau_{1} \searrow \sigma_{2} \nearrow \tau_{2} \searrow \cdots \searrow \sigma_{k-1} \nearrow \tau_{k-1} \searrow \sigma_{k}
$$

be an alternating chain of horizontal simplices. Then $\sigma_{1} \cup \sigma_{2} \cup \cdots \cup \sigma_{k}$ is a simplex.

Proof. First, we use induction to show that $\sigma_{1} \cup \sigma_{k}$ is a simplex. The case of a length two chain

$$
\sigma_{1} \nearrow \tau_{1} \searrow \sigma_{2}
$$

is obvious. For longer chains, we can use the transitivity of $\searrow$ and the Shortening Lemma (5.14) to argue that

$$
\sigma_{1} \nearrow\left(\sigma_{1} \cup \tau_{2}\right) \searrow \sigma_{3} \nearrow \tau_{3} \searrow \cdots \searrow \sigma_{k-1} \nearrow \tau_{k-1} \searrow \sigma_{k}
$$

or

$$
\sigma_{1} \nearrow \tau_{1} \searrow \sigma_{3} \nearrow \tau_{3} \searrow \cdots \searrow \sigma_{k-1} \nearrow \tau_{k-1} \searrow \sigma_{k}
$$

is a shorter alternating chain from $\sigma_{1}$ to $\sigma_{k}$, whence $\sigma_{1} \cup \sigma_{k}$ is a simplex by induction hypothesis.

Now, we apply this argument to subsequences

$$
\sigma_{i} \nearrow \tau_{i} \searrow \sigma_{i+1} \nearrow \tau_{i+1} \searrow \cdots \searrow \sigma_{j-1} \nearrow \tau_{j-1} \searrow \sigma_{j}
$$

and find that $\sigma_{i} \cup \sigma_{j}$ is a simplex for any two indices, $i$ and $j$. Since the Euclidean building $X$ is a flag complex, it follows that $\sigma_{1} \cup \sigma_{2} \cup \cdots \cup \sigma_{k}$ is a simplex.

q.e.d.

Proof of Proposition (5.4). By Lemma (5.16), for any strictly alternating chain there is a simplex that contains its lower terms (i.e., the elements to which the move is going down or from where the move is going up). This simplex has at most $2^{\operatorname{dim}(X)+1}-1$ faces. Since Corollary (5.15) rules out any repetitions in a chain, the length of any strictly alternating chain is therefore 
bounded by $2\left(2^{\operatorname{dim}(X)+1}-1\right)+1$ which accounts for a possible move down in the beginning and a move up at the end.

Also note that the longest possible sequences of moves going down have length $\leq \operatorname{dim}(X)$, and there are no $\nearrow$-chains of length 2 or longer by Lemma (5.6).

It follows that we can take the uniform upper bound to be $\operatorname{dim}(X)\left(2\left(2^{\operatorname{dim}(X)+1}-1\right)+1\right)$.

q.e.d.

\section{Descending Links: the Irreducible Case}

We retain hypotheses, notation, and terminology from the previous section. In particular, the Euclidean building $X$ is still assumed to be irreducible.

We subdivide $X$ as follows. Each horizontal simplex is barycentrically subdivided. Note that any simplex can be written as the join of its maximal horizontal faces. Thus, each simplex has an induced subdivision. Also note that the subdivision rule is compatible with face relations. Thus, we have defined a subdivision of $X$, which we will denote by $X^{\circ}$. Note that the vertices of ${ }^{X}$ are in 1-1-correspondence with the horizontal simplices of $X$. We denote by $\stackrel{\circ}{\tau}$ the vertex in $\stackrel{\circ}{X}$ corresponding to the horizontal simplex $\tau$ in $X$. Simplices in $\stackrel{\circ}{X}$ correspond to sets of chains

$$
\left\{\tau_{1}^{1}<\tau_{2}^{1}<\cdots<\tau_{k_{1}}^{1}=: \tau^{1}, \ldots, \tau_{1}^{l}<\tau_{2}^{l}<\cdots<\tau_{k_{l}}^{l}=: \tau^{l}\right\}
$$

where $\tau^{1}, \tau^{2}, \ldots, \tau^{l}$ are horizontal faces (of different $\beta$-heights) of a common simplex $\tau$. We infer:

Observation 6.1. The link of a vertex $\stackrel{\circ}{\epsilon} \stackrel{\circ}{X}$ decomposes as a join

$$
\operatorname{Lk}(\stackrel{\circ}{\tau})=\operatorname{Lk}_{\partial}(\stackrel{\circ}{\tau}) * \operatorname{Lk}_{\delta}(\stackrel{\circ}{\tau})
$$

where the face part $\operatorname{Lk}_{\partial}(\stackrel{\circ}{)})$ is the barycentric subdivision of the boundary $\partial(\tau)$ and the coface part $\operatorname{Lk}_{\delta}(\stackrel{\circ}{\tau})$ is $\operatorname{Lk}(\tau) \subseteq X$ with the induced subdivision.

q.e.d.

Observe that $\beta$ and dp are well-defined on vertices of $\dot{X}$. Also, each vertex $\stackrel{\circ}{\tau}$ (corresponding to the horizontal simplex $\tau$ ) has a dimension $\operatorname{dim}(\stackrel{\circ}{\tau}):=$ $\operatorname{dim}(\tau)$. We define the Morse function

$$
\begin{aligned}
h: \stackrel{\circ}{X} & \longrightarrow \mathbb{R} \times \mathbb{R} \\
\stackrel{\circ}{\tau} & \mapsto(\beta(\stackrel{\circ}{\tau}),(\operatorname{dim}(X)+1) \operatorname{dp}(\stackrel{\circ}{\tau})+\operatorname{dim}(\stackrel{\circ}{\tau}))
\end{aligned}
$$


In order to meaningfully talk about its sublevel sets, we need to endow $\mathbb{R} \times \mathbb{R}$ with an order relation. We do so by lexicographic order, i.e., $\left(s_{1}, s_{2}\right) \leq\left(t_{1}, t_{2}\right)$ if and only if

$$
s_{1}<t_{1}
$$

or

$$
s_{1}=t_{1} \quad \text { and } \quad s_{2} \leq t_{2} .
$$

In other words, if $\beta$ decides, we follow that decision; but if $\beta$ yields a tie, we use dp to break it; and if dp still does not allow us to make a decision, we resort to dim.

Observation 6.2. Let $\stackrel{\circ}{\tau}_{1}$ and $\stackrel{\circ}{\tau}_{2}$ be two vertices in $\stackrel{\circ}{X}$ (corresponding to the horizontal simplices $\tau_{1}$ and $\left.\tau_{2}\right)$. Suppose $\stackrel{\circ}{\tau}_{1}$ and $\stackrel{\circ}{\tau}_{2}$ span an edge. Then either $\beta\left(\stackrel{\circ}{\tau}_{1}\right) \neq \beta\left(\stackrel{\circ}{\tau}_{2}\right)$ or $\tau_{1}$ and $\tau_{2}$ are nested, i.e., one is a face of the other. Note that in this case, $\operatorname{dim}\left(\stackrel{\circ}{\tau}_{1}\right) \neq \operatorname{dim}\left(\stackrel{\circ}{2}_{2}\right)$. Consequently, there are no h-horizontal edges in $X$.

q.e.d.

Observation 6.3. Note that $(\operatorname{dim}(X)+1) \operatorname{dp}(\tau)+\operatorname{dim}(\tau)$ is uniformly bounded from above by a constant, say, $N$. Then the sublevel complex in $\stackrel{\circ}{X}$ spanned by the vertex set $\{\stackrel{\circ}{\tau} \mid h(\stackrel{\circ}{\tau}) \leq(r, N)\}$ is a subdivision of the sublevel complex in $X$ spanned by the vertex set $\{v \mid \beta(v) \leq r\}$. In particular, both sublevel complexes have the same connectivity.

q.e.d.

Since we put have an order on the range $\mathbb{R} \times \mathbb{R}$ of the Morse function, we can define descending links $\operatorname{Lk}^{\downarrow}(\stackrel{\circ}{\tau})$ as usual as the part of the link arising from those cells that contain $\stackrel{\circ}{\tau}$ as their unique highest vertex.

Observation 6.4. The Euclidean building $X$ is a flag complex and so is the subdivision $\dot{X}$. It follows that the links $\operatorname{Lk}(\stackrel{\circ}{\tau})$ are flag complexes, too. The descending link $\mathrm{Lk}^{\downarrow} \stackrel{\circ}{\tau}$ is therefore the subcomplex spanned by all adjacent vertices in $\stackrel{\circ}{X}$ of strictly smaller h-height.

It follows that the descending links inherits a decomposition from the link as a join parts

$$
\operatorname{Lk}^{\downarrow}(\stackrel{\circ}{\tau})=\operatorname{Lk}_{\partial}^{\downarrow}(\stackrel{\circ}{\tau}) * \operatorname{Lk}_{\delta}^{\downarrow}(\stackrel{\circ}{\tau})
$$

where $\operatorname{Lk}_{\partial}^{\downarrow}(\stackrel{\circ}{\tau}):=\operatorname{Lk}^{\downarrow}(\stackrel{\circ}{\tau}) \cap \operatorname{Lk}_{\partial}(\stackrel{\circ}{\tau})$ and $\operatorname{Lk}_{\delta}^{\downarrow}(\stackrel{\circ}{\tau}):=\operatorname{Lk}^{\downarrow}(\stackrel{\circ}{\tau}) \cap \operatorname{Lk}_{\delta}(\stackrel{\circ}{\tau})$. $\quad$ q.e.d.

Lemma 6.5. Let $\tau$ be a horizontal simplex with $\tau^{\min } \neq \tau$. Then $\operatorname{Lk}^{\downarrow}(\stackrel{\circ}{\tau})$ is contractible. 
Proof. Since the descending link decomposes as a join $\operatorname{Lk}^{\downarrow}\left({ }^{\circ}\right)=\operatorname{Lk}_{\partial}^{\downarrow}(\stackrel{\circ}{\tau}) *$ $\operatorname{Lk}_{\delta}^{\downarrow}(\tau)$, it suffices to show that the descending face part $\mathrm{Lk}_{\partial}^{\downarrow}(\stackrel{\circ}{\tau})$ is contractible. Recall that the face part $\operatorname{Lk}_{\partial}(\stackrel{\circ}{\tau})$ is just the barycentric subdivision of the sphere $\partial(\tau)$.

Since $\tau^{\min } \neq \tau$, we have $\tau^{\min } \nearrow \tau$ whence $\operatorname{dp}\left(\tau^{\min }\right)>\operatorname{dp}(\tau)$. Consequently, $\operatorname{Lk}_{\partial}^{\downarrow}(\tau)$ misses the vertex $\tau^{\text {min }}$.

On the other hand, for any proper face $\sigma<\tau$ with $\tau^{\min } \not \leq \sigma$ we have $\tau \searrow \sigma$, whence $\operatorname{dp}(\sigma)<\operatorname{dp}(\tau)$, i.e., the descending face part contains all vertices $\sigma$ for $\tau^{\min } \not \leq \sigma<\tau$.

Note that we cannot say anything about the depth of simplices $\sigma$ with $\tau^{\min }<\sigma<\tau$. Nonetheless, the information we have is enough to deduce that $\operatorname{Lk}_{\partial}^{\downarrow}(\tau)$ is homotopy equivalent to a once-punctures sphere and hence contractible: Let $B$ be the subcomplex of $\operatorname{Lk}_{\partial}(\stackrel{\circ}{\tau})$ spanned by the set $\left\{\stackrel{\circ}{\sigma} \tau^{\min } \not \leq \sigma<\tau\right\}$. The geometric realization of $B$ is the sphere $\partial(\tau)$ with the open star of the simplex $\tau^{\min }$ removed. Thus, $B$ is a closed ball and hence contractible. We have seen that $B$ is a subcomplex of $\operatorname{Lk}_{\partial}^{\downarrow}(\tau)$. Projecting away from $\tau^{\mathrm{min}}$ defines a deformation retraction from $\operatorname{Lk}_{\partial}^{\downarrow}(\stackrel{\circ}{\tau})$ onto $B$. Hence $\operatorname{Lk}_{\partial}^{\downarrow}(\stackrel{\sim}{\tau})$ is contractible.

q.e.d.

Lemma 6.6. Let $\tau$ be a horizontal simplex $\tau$ that satisfies $\tau=\tau^{\min }$. If $X$ is thick, then $\operatorname{Lk}^{\downarrow}(\stackrel{\sim}{\tau})$ is $(\operatorname{dim}(X)-2)$-connected.

Proof. Again, we use the decomposition $\operatorname{Lk}^{\downarrow}(\stackrel{\circ}{\tau})=\operatorname{Lk}_{\partial}^{\downarrow}(\stackrel{\circ}{\tau}) * \operatorname{Lk}_{\delta}^{\downarrow}(\stackrel{\circ}{\tau})$. First note that for each proper face $\sigma<\tau$, we have $\tau=\tau^{\min } \not \leq \sigma<\tau$, i.e., $\tau \searrow \sigma$. Hence, $\operatorname{dp}(\sigma)<\operatorname{dp}(\tau)$. It follows that $\operatorname{Lk}_{\partial}^{\downarrow}(\tau)=\operatorname{Lk}_{\partial}(\stackrel{\sim}{\tau})$, which is homeomorphic to the sphere $\partial(\tau)$.

We now have to understand the descending coface part $\operatorname{Lk}_{\delta}^{\downarrow}(\tau)$. Recall that $\operatorname{Lk}_{\delta}(\tau)$ is just a subdivision of $\operatorname{Lk}(\tau)=\operatorname{Lk}_{\text {hor }}(\tau) * \operatorname{Lk}_{\text {ver }}(\tau)$ where the subdivision of a simplex $\sigma:=\sigma_{\text {hor }} * \sigma_{\text {ver }} \subset \operatorname{Lk}_{\text {hor }}(\tau) * \operatorname{Lk}_{\text {ver }}(\tau)$ is induced by the barycentric subdivisions of all its maximal horizontal faces. In particular, if $\sigma_{\text {ver }}$ does not contain any equatorial vertices then $\sigma_{\text {hor }}$ is a maximal horizontal face and the subdivision of $\sigma$ is given as the join of the subdivisions of $\sigma_{\text {hor }}$ and $\sigma_{\text {ver }}$.

In general, $\sigma_{\text {ver }}$ might contain equatorial vertices. In that case, the subdivision of $\sigma=\sigma_{\text {hor }} * \sigma_{\text {ver }}$ might not naturally split as a join of a vertical and a horizontal part. However, we shall see that under the assumption $\tau=\tau^{\min }$, the descending coface part $\operatorname{Lk}_{\delta}^{\downarrow}(\tau)$ does decompose as a join of a horizon- 
tal and a vertical component: each simplex will factor as described in the previous paragraph.

The key observation is that the descending link does not contain any equatorial barycenters from the vertical link $\operatorname{Lk}_{\mathrm{ver}}(\tau)$, provided $\tau^{\min }=\tau$. To see this, consider a horizontal coface $\xi$ of $\tau$ so that $\xi \backslash \tau$ does not contribute to the horizontal link. By Lemma (5.2), this means $\xi^{\min } \not \leq \tau$, which implies $\xi \searrow \tau$, whence $\operatorname{dp}(\xi)>\operatorname{dp}(\tau)$. Thus, $\xi$ is not in the descending link of $\stackrel{\tau}{\tau}$.

With this decomposition of $\operatorname{Lk}_{\delta}^{\downarrow}(\tau)$, we are ready to determine its connectivity. The horizontal link $\operatorname{Lk}_{\text {hor }}(\tau)$ (barycentrically subdivided) is fully descending. For each horizontal coface $\xi$ with $\xi^{\min } \leq \tau<\xi$, we have $\xi^{\min }=\tau^{\min }=\tau$ by Observation (5.3). Thus, $\tau \nearrow \xi$, whence $\operatorname{dp}(\xi)<\operatorname{dp}(\tau)$. Thus, $\stackrel{\xi}{\xi} \in \mathrm{Lk}_{\delta}^{\downarrow}(\stackrel{\circ}{\tau})$.

Finally, we consider the vertical part of the descending link of $\tau$. Since we have already seen that no equatorial simplices of $\operatorname{Lk}_{\mathrm{ver}}(\tau)$ contribute to $\operatorname{Lk}^{\downarrow}(\stackrel{\circ}{\tau})$, we see that the Busemann function $\beta$ decides which vertices contribute. More precisely, let $\sigma$ be a horizontal simplex in $\operatorname{Lk}_{\text {ver }}(\tau)$, then $\stackrel{\circ}{\sigma} \in \operatorname{Lk}_{\delta}^{\downarrow}(\stackrel{\circ}{\tau})$ if and only if $\beta(\sigma)<\beta(\tau)$. It follows that $\operatorname{Lk}_{\delta}^{\downarrow}(\tau)$ is a subdivision of an open hemisphere complex $H$ in the vertical link $\operatorname{Lk}_{\mathrm{ver}}(\tau)$ induced by the Busemann gradient. Since we used that gradient to separate the vertical and the horizontal factors in $\operatorname{Lk}(\tau)=\operatorname{Lk}_{\text {hor }}(\tau) * \operatorname{Lk}_{\mathrm{ver}}(\tau)$, it follows from Theorem (4.3) that the open hemisphere complex $\operatorname{Lk}_{\mathrm{ver}}^{\downarrow}(\tau)$ is spherical of dimension $\operatorname{dim}\left(\operatorname{Lk}_{\mathrm{ver}}(\tau)\right)$.

Thus,

$$
\operatorname{Lk}^{\downarrow}(\stackrel{\circ}{\tau}) \partial(\tau) * \operatorname{Lk}_{\text {hor }}(\tau) * H
$$

is spherical of dimension $\operatorname{dim}(X)-1$, hence $(\operatorname{dim}(X)-2)$-connected. q.e.d.

\section{Descending Links: the General Case}

Now, we finally drop the irreducibility hypothesis. However, we add the assumption of thickness. Let

$$
X=X_{1} \times \cdots \times X_{k}
$$

be a thick Euclidean building written as a product of thick irreducible Euclidean buildings $X_{i}$. Suppose we are given a function

$$
\begin{aligned}
\beta: X & \longrightarrow \mathbb{R} \\
\left(x_{1}, \ldots, x_{k}\right) & \mapsto \sum_{i} a_{i} \beta_{i}\left(x_{i}\right)
\end{aligned}
$$


as a positive $\left(a_{i}>0\right)$ linear combination of Busemann functions $\beta_{i}: X_{i} \rightarrow \mathbb{R}$. We subdivide all $X_{i}$ as in Section 6 and put

$$
\stackrel{\circ}{X}:=\stackrel{\circ}{X}_{1} \times \cdots \times \stackrel{\circ}{X}_{k} .
$$

Note that $\stackrel{\circ}{X}$ is a poly-simplicial complex, i.e., each cell is a product of simplices. In particular, we can regard $\dot{X}$ as a piecewise Euclidean complex.

Also, we extend $\beta$ to a Morse function

$$
\begin{aligned}
h: \stackrel{\circ}{X} & \longrightarrow \mathbb{R} \times \mathbb{R} \\
\left(\stackrel{\circ}{\tau}_{1}, \ldots, \stackrel{\circ}{\tau}_{k}\right) & \mapsto\left(\sum_{i} a_{i} \beta_{i}\left(\tau_{i}\right), \sum_{i}\left(\operatorname{dim}\left(X_{i}\right)+1\right) \operatorname{dp}_{i}\left(\tau_{i}\right)+\operatorname{dim}\left(\tau_{i}\right)\right)
\end{aligned}
$$

Note that edges in $\stackrel{\circ}{X}^{\prime}=\stackrel{\circ}{X}_{1} \times \cdots \times \stackrel{\circ}{X}_{k}$ always arise from an edge in a single factor. Therefore, there are no edges in $\stackrel{\circ}{X}$ horizontal with respect to $h$.

The descending link $\mathrm{Lk}^{\downarrow}(\stackrel{\circ}{\tau})$ of a vertex $\stackrel{\circ}{\tau} \in \stackrel{\circ}{X}$ is defined as the subcomplex of the link $\operatorname{Lk}(\stackrel{\circ}{\tau})$ induced by all those poly-simplices $\tau$ containing $\stackrel{\circ}{\tau}$ as the unique point in $\tau$ where $h$ is maximal. Since addition

$$
\sum:(\mathbb{R} \times \mathbb{R})^{k} \longrightarrow \mathbb{R} \times \mathbb{R}
$$

is strictly monotonic in each of the $k$ arguments, we deduce the following two observations, the first of which strengthens slightly the statement that there are no $h$-horizontal edges.

Observation 7.1. Every cell in $\stackrel{\circ}{X}$ has a unique h-highest vertex.

The second observation nails the structure of descending links. They decompose as joins of descending links taken in the factors $\dot{X}_{i}$.

Observation 7.2. For each vertex $\stackrel{\circ}{\tau}=\left(\stackrel{\circ}{\tau}_{1}, \stackrel{\circ}{\tau}_{2}, \ldots, \stackrel{\circ}{\tau}_{k}\right) \in \stackrel{\circ}{X}$, we have

$$
\operatorname{Lk}^{\downarrow}(\stackrel{\circ}{\tau})=\operatorname{Lk}^{\downarrow}\left(\stackrel{\circ}{\tau}_{1}\right) * \operatorname{Lk}^{\downarrow}\left(\stackrel{\circ}{\tau}_{2}\right) * \cdots * \operatorname{Lk}^{\downarrow}\left(\stackrel{\circ}{\tau}_{k}\right)
$$

Remark 7.3. In the more general case where we allow some of the coefficient $a_{i}$ to vanish, the descending links in $\stackrel{\circ}{X}$ are joins of descending links in those $\stackrel{\circ}{X}_{i}$ where $a_{i} \neq 0$.

Proposition 7.4. For any vertex $\stackrel{\circ}{\tau}=\left(\stackrel{\circ}{\tau}_{1}, \ldots, \stackrel{\circ}{\tau}_{k}\right) \in \stackrel{\circ}{X}$, the descending link $\mathrm{Lk}^{\downarrow}(\stackrel{\circ}{\tau})$ is $(\operatorname{dim}(X)-2)$-connected. 
Proof. We have $\operatorname{Lk}^{\downarrow}(\stackrel{\circ}{\tau})=\operatorname{Lk}^{\downarrow}\left(\stackrel{\circ}{\tau}_{1}\right) * \operatorname{Lk}^{\downarrow}\left(\stackrel{\circ}{2}_{2}\right) * \cdots * \operatorname{Lk}^{\downarrow}\left(\stackrel{\circ}{\tau}_{k}\right)$. By Lemmata (6.5) and (6.6), the factor $\operatorname{Lk}^{\downarrow}\left(\tau_{i}\right)$ is $\left(\operatorname{dim}\left(X_{i}\right)-2\right)$-connected. The claim now follows since the join of an $m$-connected space and an $n$-connected space is $(m+n+2)$-connected.

q.e.d.

We shall now adapt Morse theory to our situation. In order to do so, we pass to a subdivision once more.

Lemma 7.5. Let $Y$ be a piecewise Euclidean complex with a map of its vertices into an ordered set so that each cell has a unique highest vertex. Then $Y$ has a simplicial subdivision that (a) does not introduce new vertices, (b) does not change the homotopy type of sublevel complexes, and (c) does not change the homotopy type of descending links.

Proof. We proceed by induction on skeleta. We do not need to subdivide the 1-skeleton. So assume that the $(n-1)$-skeleton is already subdivided. To subdivide an $n$-cell, cone off the subdivision of its boundary from the unique top vertex. It is clear that this subdivision rule does not introduce new vertices. Since we used the top vertex as the cone point, it also does not change (but subdivides) sublevel sets and descending links.

q.e.d.

Going back to the special situation at hand, recall how in the the proof of Proposition (4.4), the main point was to find a number $\varepsilon$ so that for each level $r$,

$$
\beta^{-1}((-\infty, r]) \hookrightarrow \beta^{-1}((-\infty, r+\varepsilon])
$$

induces isomorphisms in homotopy groups $\pi_{n}$ for $n \leq \operatorname{dim}(X)-2$. Back there, we noted that by Observation (4.6), there is an $\varepsilon$ so that $\beta^{-1}([r, r+\varepsilon])$ does not contain complete edges. In the presence of horizontal edges, this is blatantly false. However, it still follows by the same argument that we can choose $\varepsilon$, independent of $r$, so that every edge contained in $\beta^{-1}([r, r+\varepsilon])$ must be horizontal. For this $\varepsilon$, we have:

Lemma 7.6. For each level $r \in \mathbb{R}$, the inclusion

$$
\beta^{-1}((-\infty, r]) \hookrightarrow \beta^{-1}((-\infty, r+\varepsilon])
$$

induces isomorphisms in homotopy groups $\pi_{n}$ for $n \leq \operatorname{dim}(X)-2$. 
Proof. First, we replace $X$ by its subdivision $\stackrel{\circ}{X}$. Note that this does not affect sublevel sets. Second, let $\stackrel{\circ}{X}_{r}$ be the subcomplex of $\stackrel{\circ}{X}^{\circ}$ spanned by all vertices in the sublevel set $\beta^{-1}((-\infty, r])$ and note that $\stackrel{\circ}{X}_{r}$ is a deformation retract of the sublevel set (by pushing in free faces). Thus, it suffices to show that the inclusion $\stackrel{\circ}{X}_{r} \hookrightarrow \stackrel{\circ}{X}_{r+\varepsilon}$ induces isomorphisms in homotopy groups $\pi_{n}$ for $n \leq \operatorname{dim}(X)-2$.

We shall use regular Bestvina-Brady Morse theory to accomplish this remaining task. We use Observation (7.1) and Lemma (7.5) to simplicially subdivide $\stackrel{\circ}{X}$ without changing descending links or sublevel sets and without introducing new vertices. Since nothing changed, we will keep the notation $\stackrel{\circ}{X}$.

Now, define a new height function on the sublevel complex $\stackrel{\circ}{X}_{r+\varepsilon}$ as follows

$$
\begin{array}{rlrl}
\tilde{h}: \stackrel{\circ}{X+\varepsilon}_{r} & \longrightarrow \mathbb{Z} \subset \mathbb{R} & \\
\stackrel{\circ}{\tau} & \mapsto \begin{cases}-1 & \text { if } \dot{\tau} \in \dot{X}_{r} \\
\sum_{i}\left(\operatorname{dim}\left(X_{i}\right)+1\right) \operatorname{dp}_{i}\left(\tau_{i}\right)+\operatorname{dim}\left(\tau_{i}\right) & \text { otherwise }\end{cases}
\end{array}
$$

Note that every vertex $\stackrel{\circ}{\tau} \in \stackrel{\circ}{X}_{r+\varepsilon} \backslash \stackrel{\circ}{X}_{r}$ has the same descending link with respect to $\tilde{h}$ as it has with respect to $h$. To see this let $\stackrel{\sigma}{\text { be a vertex in }}$ the link of $\stackrel{\circ}{\tau}$. If $\stackrel{\circ}{\sigma}$ also belongs to $\stackrel{\circ}{X}_{r+\varepsilon} \backslash \stackrel{\circ}{X}_{r}$ the edge connecting $\stackrel{\circ}{\sigma}$ and $\stackrel{\circ}{\tau}$ is $\beta$-horizontal by our choice of $\varepsilon$. It follows that whether $\stackrel{\circ}{\sigma}$ is descending is determined by the secondary Morse function. If $\stackrel{\circ}{\sigma} \in \stackrel{\circ}{X}$, it is clearly descending with respect to both Morse functions.

We put $\stackrel{\circ}{X}(n):=\tilde{h}^{-1}([-1, n])$. Note that $\stackrel{\circ}{X}_{r}=\stackrel{\circ}{X}(-1)$ and $\stackrel{\circ}{X}_{r+\varepsilon}=\stackrel{\circ}{X}(n)$ for large $n$. By [BeBr97, Lemma 2.5], passing from $\dot{X}^{\circ}(n)$ to $\dot{X}(n+1)$ changes the homotopy type exactly by coning off descending links of all vertices $\stackrel{\sim}{\tau}$ with $\tilde{h}(\stackrel{\circ}{\tau})=n+1$. By Proposition (7.4), descending links are $(\operatorname{dim}(X)-2)$ connected and the claim follows.

q.e.d.

We obtain the following theorem as an easy corollary:

Theorem 7.7. Let $X=X_{1} \times \cdots \times X_{k}$ be a thick Euclidean building written as a product of irreducible Euclidean buildings. Then the spherical building at infinity decomposes as a join $\partial^{\infty}(X)=\partial^{\infty}\left(X_{1}\right) * \cdots * \partial^{\infty}\left(X_{k}\right)$. Thus, we can think of points in $\partial^{\infty}(X)$ as convex linear combinations of points in the $\partial^{\infty}\left(X_{i}\right)$. Let $e_{\infty} \in \partial^{\infty}(X)$ be a point at infinity with non-trivial coordinates in each $\partial^{\infty}\left(X_{i}\right)$. Equivalently, assume that $e_{\infty}$ is not contained in any subspace $\partial^{\infty}\left(X_{1}\right) * \cdots * \partial^{\infty}\left(X_{i-1}\right) * \partial^{\infty}\left(X_{i+1}\right) * \cdots * \partial^{\infty}\left(X_{k}\right)$. Then complements of horoballs centered at $e_{\infty}$ are $(\operatorname{dim}(X)-2)$-connected. 
Proof. The proof is the same as in the final steps of proving Proposition (4.4): By Lemma (7.6), the inclusion

$$
\beta^{-1}((-\infty, r]) \hookrightarrow \beta^{-1}((-\infty, r+\varepsilon]) \hookrightarrow \beta^{-1}((-\infty, r+2 \varepsilon]) \hookrightarrow \cdots \hookrightarrow X
$$

induces isomorphisms in $\pi_{n}$ for $n \leq \operatorname{dim}(X)-2$. Since $X$ is contractible, it follows that sublevel sets are $(\operatorname{dim}(X)-2)$-connected.

q.e.d.

Using Remark (7.3), the same argument shows:

Theorem 7.8. Let $\beta=\sum_{i} a_{i} \beta_{i}: X \rightarrow \mathbb{R}$ be a non-negative linear combination of Busemann functions. Then, level- and sublevel-set of $\beta$ in $X$ are $\left(\left(\sum_{a_{i} \neq 0} \operatorname{dim}\left(X_{i}\right)\right)-2\right)$-connected.

We note that the thickness hypothesis in our connectivity results derives entirely from the use of Theorem (4.3) via Lemma (6.6) and the assumption could be dropped here if it could be removed from Schulz' result.

We remark that the results of this section can be used to determine geometric invariants of actions of certain $S$-arithmetic groups on their associated symmetric spaces in the number field case [Rehn07. We also note that a generalization of our results to $\mathbb{R}$-buildings, which arise, e.g., as asymptotic cones, would be of interest.

\section{Finitness Properties of Rank One Groups}

We are now in a position to prove Theorem [1.2. Recall that $K$ is a global function field, $\mathbf{G}$ is a noncommutative, absolutely almost simple $K$-group of $K$-rank 1 , and $S$ is a finite set of pairwise inequivalent valuations on $K$. We can restate Theorem 1.2 as follows:

Theorem 8.1. The $S$-arithmetic group $\mathbf{G}\left(\mathcal{O}_{S}\right)$ is of type $F_{m-1}$ where $m:=$ $\sum_{v \in S} \operatorname{rank}_{K_{v}}(\mathbf{G})$ is the sum of local ranks or equivalently, the dimension of the associated Euclidean building $X:=\prod_{v \in S} X_{v}$.

Proof. Let $\mathcal{H}$ be a collection of closed horoballs as in Theorem 3.7. By further increasing the parameter $d$ used to define $\mathcal{H}$, we can choose the horoballs so that the distance between any two exceeds the diameter of the polyhedral cells in $X$. With this choice, every cell of $X$ meets at most one horoball. It 
follows that we can subdivide $X$ so that all horoballs in $\mathcal{H}$ become subcomplexes of the CW-complex $X$.

Let $Y:=X \backslash \bigcup_{H \in \mathcal{H}} \stackrel{\circ}{H}$ denote the complement of the open horoballs. This is a CW-subcomplex of $X$ containing the horospheres $\partial(H)$. By Theorem 3.7 , the group $\mathrm{G}\left(\mathcal{O}_{S}\right)$ acts cocompactly on $Y$. Cell stabilizers are finite. By [Bro87, Propositions 1.1 and 3.1], it suffices to show that $Y$ is $(m-2)$ connected.

We have seen in Theorem [7.7, that each horosphere $\partial(H)$ is $(m-2)$ connected. Let $Z$ denote $Y$ with the horospheres in $\{\partial(H) \mid H \in \mathcal{H}\}$ collapsed. Collapsing disjoint $n$-connected subcomplexes independently does not affect homotopy groups in dimensions up to $n$. It follows that $\pi_{n}(Y)=$ $\pi_{n}(Z)$ for $n \leq m-2$.

On the other hand, $Z$ can also be obtained from $X$ by collapsing independently the horoballs $H \in \mathcal{H}$. Since collapsing contractible subcomplexes does not affect the homotopy type, we deduce that $Z$ is contractible. Hence $Y$ is $(m-2)$-connected.

q.e.d.

\section{References}

[Abel91] H.Abels: Finiteness Properties of certain arithmetic groups in the function field case. Israel J. Math. 76 (1991) p. 113 - 128

[Abra96] P. Abramenko: Twin Buildings and Applications to S-Arithmetic Groups. Springer LNM 1641 (1996)

[Behr69] H. Behr: Endliche Erzeugbarkeit arithmetischer Gruppen über Funktionenkörpern. Inventiones mathematicae 7 (1969) p. 1 - 32

[BeBr97] M. Bestvina, N. Brady: Morse theory and finiteness properties of groups. Inventiones mathematicae 129 (1997) p. 445 - 470

[Bro87] K.S. Brown: Finiteness Properties of Groups. Journal of Pure and Applied Algebra 44 (1987) p. $45-75$

[BuGo99] K-U.Bux, C.R. Gonzalez: The Bestvina-Brady Construction Revisited - Geometric Computation of $\Sigma$-Invariants for Right Angled Artin Groups. Journal of the London Mathematical Society (2) 60 (1999) p. $793-801$ 
[BW07] K.-U.Bux, K. Wortman: Finiteness properties of arithmetic groups over function fields. Inventiones mathematicae 167 (2007) p. $355-378$

[Hard69] G. Harder: Minkowskische Reduktionstheorie über Funtionenkörpern. Inventiones mathematicae 7 (1969) p. 33 - 54

[Marg91] G.A. Margulis: Discrete Subgroups of Semisimple Lie Groups. Ergebnisse der Mathematik 3. Folge, vol. 17 (Springer, 1991)

[Ragh72] M.S. Raghunathan: Discrete subgroups of Lie Groups. Ergebnisse der Mathematik und ihrer Grenzgebiete 68, Springer New YorkHeidelberg (1972)

[Rehn07] W.H. Rehn: Kontrollierter Zusammenhang über symmetrischen Räumen. PhD thesis (Frankfurt am Main, 2007)

[Schu05] B. Schulz: Sphärische Unterkomplexe sphärischer Gebäude. PhD thesis (Frankfurt am Main, 2005)

[Spri94] T.A.Springer: Reduction theory over global fields. Proc. Indian Acad. Sci. Math. Sci. 104 (1994) p. 207 - 216

[Stuh80] U.Stuhler: Homological properties of certain arithmetic groups in the function field case. Inventiones mathatematicae 57 (1980) p. $263-281$ 